\title{
Characterization of selective etching and patterning by sequential light- and heavy-ion irradiation of $\mathrm{LiNbO}_{3}$
}

Hsu-Cheng Huang ${ }^{1}$, Girish Malladi ${ }^{2}$, Lihua Zhang ${ }^{3}$, Jerry I. Dadap ${ }^{1}$, Kim Kisslinger ${ }^{3}$, Hassaram Bakhru ${ }^{2}$, and Richard M. Osgood, Jr. ${ }^{1}$

\footnotetext{
${ }^{(1)}$ Center for Integrated Science \& Engineering, Columbia University, New York, New York 10027, United States

${ }^{(2)}$ College of Nanoscale Science and Engineering, SUNY Polytechnic Institute, Albany, NY 12203, United States

${ }^{(3)}$ Center for Functional Nanomaterials, Brookhaven National Laboratory, Upton, New York 11973, United States
}

\begin{abstract}
The induced selective etching properties of $\mathrm{LiNbO}_{3}$ in a sample subjected to ion processing using sequential light- and heavy-ion irradiation are investigated and discussed. Through the use of TEM and SEM, the lattice structure at the amorphous-crystalline interface is examined after heavy ion exposure and it is found that single-energy amorphizing irradiation results in undercut etching at the interface, while multiple-energy irradiation yields sharper features. Such sequential-irradiation process based on both light- and heavy-ion irradiation enables ready fabrication of concomitant high-resolution patterning and exfoliation of structured freestanding thin films.
\end{abstract}

\section{Introduction}

Lithium niobate $\left(\mathrm{LiNbO}_{3}\right)$ is widely used in a variety of microsystem applications due to its outstanding characteristics, including optoelectronic, optomechanical, and nonlinear-optical 
properties. The employment of these properties has generated a number of potential $\mathrm{LiNbO}_{3}$ devices for current and future photonics systems [1]. For many of these applications, integrated devices with planar-processed and patterned thin films are crucial for precise control of optical signals. An impediment to patterning $\mathrm{LiNbO}_{3}$ for such devices is the fact that this metal-oxide crystal is known to be a highly chemically resistant material [2] and this chemical inertness limits the ability to fabricate high-aspect-ratio structures and, in many cases, reduces the possibilities for materials-selective etching. In addition, the importance of thin-film forms for integrated structures has led to new approaches to fabricating those films. One approach, ion slicing, has used light implantation of high-energy ions followed by selective etching of the buried damage region. The employment of both of these methods is an attractive approach for microstructure formation in such relatively inert oxide. However, while there have been numerous studies of the materials physics of light-ion induced materials change, including those directed toward ion-slicing of oxides, much less is known regarding the effect of bombardment by heavy ions or a combination of both light and heavy ions on such oxide materials.

As an example of recent studies on heavy ion physics, broad-area ion-beam enhanced etching (IBEE) of $\mathrm{LiNbO}_{3}$ using electronic-excitation damage from heavy-ion irradiation has been proposed and demonstrated as a possible alternative [3, 4]. In this case, etching is enabled by the presence of high density of irradiation-induced defects, resulting in an amorphous-like damaged layer. The chemical resistance in this damaged layer, which is achieved if the irradiation dose is raised above a certain irradiation threshold, is significantly lowered from that for single-crystal $\mathrm{LiNbO}_{3}$, thus increasing the former's etch rate. For example, it was found [5] that the etching rate in 5-MeV-Si-irradiated $\mathrm{X}$-cut $\mathrm{LiNbO}_{3}$ was $\sim 100 \mathrm{~nm} / \mathrm{min}$. This radiationenabled selective etching thus has the potential for fabricating nano- or micro-structured 
photonic devices, such as ridge or one-dimensional (1D) photonic crystal waveguides [6-8]. This heavy-ion-enabled research demonstrates the potential of this etching method. However, detailed examination of the lattice structure at the interface and critical characterization of such processing parameters such as sidewall abruptness, dose dependence, etc. remains to be explored. In addition, the interaction between sequential light and heavy ion materials alterations has yet to be explored.

In this paper, we investigate the material physics of heavy-ion etching and sequential light- and heavy-ion etching through the use of transmission electron microscopy (TEM), scanning electron microscopy (SEM), and optical microscopy (OM). We first examine ioninduced modifications in atomic structure and their relation to the mechanism of ion etching. The results show that enhanced etching results from materials amorphization. In addition, the abruptness of the etched sidewall profile using single-energy amorphizing irradiation is constrained by its damage straggling distribution, while a sharper feature can be constructed using multiple-energy irradiation. We further show that the method using two sequential ion exposures, followed by a single selective-etching step, enables easy patterning of a fragile freestanding $\mathrm{LiNbO}_{3}$ thin film. The fabrication of a rib-like structure, such as is commonly used in certain optical waveguides, is demonstrated via this sequential irradiation method.

\section{Experimental}

The etching samples were congruent Z-cut single-crystal $\mathrm{LiNbO}_{3}$ (Crystal Technology). Etching was studied after bombardment by light ions, $\mathrm{He}^{+}$, and/or one of two choices of heavy ions, i.e., $\mathrm{Fe}^{+}$(reactive) and $\mathrm{Ar}^{+}$(inert) ions. For $\mathrm{He}^{+}$, beam energies of $3.6-3.8 \mathrm{MeV}$ and a dose

of $5 \times 10^{16} \mathrm{~cm}^{-2}$ was used. The irradiation was performed under a water-cooled environment, with the samples tilted $7^{\circ}$ from the beam direction to prevent channeling. For the heavy ions, beam 
energies of $350 \mathrm{keV}$ and $800 \mathrm{keV}$ at a similar dose of $1 \times 10^{15} \mathrm{~cm}^{-2}$ were used for $\mathrm{Fe}^{+}$and $\mathrm{Ar}^{+}$, respectively. SRIM [9] calculations showed that their stopping ranges $\left(R_{p}\right)$ and straggling distances $\left(\Delta \mathrm{R}_{\mathrm{p}}\right)$ in $\mathrm{LiNbO}_{3}$ were as follows: $\left(\mathrm{R}_{\mathrm{p}}, \Delta \mathrm{R}_{\mathrm{p}}\right) \approx(200 \mathrm{~nm}, 50 \mathrm{~nm})$ and $(550 \mathrm{~nm}, 150 \mathrm{~nm})$ for $\mathrm{Fe}^{+}$and $\mathrm{Ar}^{+}$, respectively. Multiple $\mathrm{Ar}^{+}$irradiation steps were also performed. The order of the irradiation steps consisted of sequential ion bombardment at energy-dose combination of $\left(800 \mathrm{keV}, 1 \times 10^{15} \mathrm{~cm}^{-2}\right),\left(525 \mathrm{keV}, 2.5 \times 10^{14} \mathrm{~cm}^{-2}\right),\left(300 \mathrm{keV}, 2.5 \times 10^{14} \mathrm{~cm}^{-2}\right),\left(150 \mathrm{keV}, 2.5 \times 10^{14}\right.$ $\left.\mathrm{cm}^{-2}\right)$, and $\left(75 \mathrm{keV}, 2.5 \times 10^{14} \mathrm{~cm}^{-2}\right)$. The etching was performed by immersing the irradiated samples in 5\% HF (hydrofluoric acid) at room temperature for eight hours.

For patterning, a blocking mask was created on a $\mathrm{LiNbO}_{3}$-sample surface prior to irradiation. The mask comprised of a photoresist (Shipley S1818) having a thickness of $2-4$ $\mu \mathrm{m}$, which was found to be sufficient to ensure that only the exposed regions were irradiated with the ion species.

For experiments involving sequential irradiation, the samples were first exposed to $\mathrm{He}^{+}$to form a deep ion-damaged sheet. Subsequently, the blocking-mask pattern was created, followed by a second heavy-ion irradiation. Note that for the sequential irradiation process, the irradiation parameters of $\mathrm{He}^{+}$and $\mathrm{Fe}^{+}$or $\mathrm{Ar}^{+}$are the same as described above. After irradiation and removal of the mask, thermal activation of the sample implantation was performed at $250^{\circ} \mathrm{C}$ for 30 minutes. The irradiated samples were then immersed in acid to investigate any etch selectivity between unirradiated and irradiated regions.

TEM specimens were prepared by in situ lift-out focused ion beam (FIB); analytical electron microscopy was performed with JEOL JEM2100F operated at $200 \mathrm{kV}$.

\section{Results and Discussion}


Consider first the effects of purely light-ion damage in $\mathrm{LiNbO}_{3}$ on wet etching, which is now a well-studied phenomenon. In light ions $\left(\mathrm{He}^{+}\right)$irradiation [10], the preponderance of ions are deposited in a relatively narrow spatial region in the target, where lattice defects are formed predominantly by Rutherford scattering (nuclear collision) around the ion stopping range. Such light-ion-induced defect networks are characterized by a concentration of twin bands or slip planes containing pileups of dislocations with helium inclusions and Frenkel pairs [11]. In $\mathrm{LiNbO}_{3}$, these defects manifest themselves as a striking series of lines at the surface arranged parallel to the threefold lattice-symmetry planes. In addition, the density of these extended defects reaches a maximum after thermally activated dislocation motion, activated during annealing at $\sim 250^{\circ} \mathrm{C}$ [12]. The existence of these local defect networks enhances an anomalously high selective etch rate $(\sim \mu \mathrm{m} / \mathrm{min})[12]$; this rate is $\sim 10^{3} \times$ greater than the etch rate in the remainder of the sample. Note that this localized selective etching enables the use of the method of crystal ion slicing [13] to fabricate freestanding thin films - with films typically of thicknesses of $2-25 \mu \mathrm{m}$. Using this method, compact optical thin-film devices have been realized, such as electro-optic prism scanners [14] and tunable poled frequency converters [15].

For purely heavy-ion-induced selective etching, the mechanisms can be anticipated to be different. Due to their large cross sections for electronic energy loss [16], ion-matter interactions are known to be much stronger throughout the full ion path [3] than for $\mathrm{H}^{+}$or $\mathrm{He}^{+}$. In particular, besides damage generated around the ion stopping range or end-of-range distance, highly cascading collisions are also formed along the heavy-ion trajectory. In fact, once the defect accumulation is sufficiently high above a certain threshold, the lattice crystallinity along the ionbeam axis becomes amorphous-like. This amorphous region can be expected to have significantly lowered chemical resistance compared with crystalline regions in the target. 
To investigate this damage phenomenon in single-crystal $\mathrm{LiNbO}_{3}, \mathrm{TEM}$ data were collected on samples, which had been irradiated with $\mathrm{Ar}^{+}$and $\mathrm{Fe}^{+}$beams. For example, Fig. 1 shows TEM images of the samples after heavy-ion irradiation only. Figure 1(a) shows a crosssectional TEM micrograph of $\mathrm{LiNbO}_{3}$ after $800 \mathrm{keV} \mathrm{Ar}{ }^{+}$irradiation at a dose of $1 \times 10^{15} \mathrm{~cm}^{-2}$; Fig. 1(b) is a zoomed-in cross-sectional image in the stopping-range region. It is clear that an amorphous-crystalline interface is seen, with a $\sim 120 \mathrm{~nm}$-thick dark-contrast transitional layer in the middle surrounded by two phases, viz., a crystalline and an amorphized region. This transitional layer corresponds to the region in which ion stopping occurs and, thus, it contains the longitudinal straggling distribution of the irradiated argon. In this region, a high density of defect clusters and their associated strain field are observed, resulting in low contrast in the image. Above the intermediate layer, selected-area diffraction patterns (SADP), as shown in Fig. 1(b) insets, reveal that the surface region is amorphous, as indicated by the characteristic diffuse ring, while the deeper unirradiated region remains crystalline, as indicated by the sharp diffraction spots. The etching profile and mechanism, especially at the interface of the amorphous and crystalline regions, are further examined and discussed in the next paragraph.

As briefly mentioned above, materials amorphization, in fact, even partial amorphization, results in enhanced etching. Therefore, with a properly designed surface mask, high-resolution patterning should be achievable. In our etching experiments, both $\mathrm{Fe}^{+}$- and $\mathrm{Ar}^{+}$-irradiated Z-cut bulk $\mathrm{LiNbO}_{3}$ samples were used first to show the efficacy of irradiation (see below for more observations using sequential implantation with light ions). In our experiments, a roomtemperature etching solution with $5 \% \mathrm{HF}$ was used. It was found that the etching rate was $20 \pm 5 \mathrm{~nm} / \mathrm{min}$ in samples irradiated with both ion species $\left(1 \times 10^{15} \mathrm{~cm}^{-2} 350-\mathrm{keV} \mathrm{Fe}^{+}\right.$or $\sim 1.5 \mathrm{dpa}$ [displacement per atom] and $1 \times 10^{15} \mathrm{~cm}^{-2} 800-\mathrm{keV} \mathrm{Ar}^{+}$or $\sim 0.7 \mathrm{dpa}$ ). This similarity in etching 


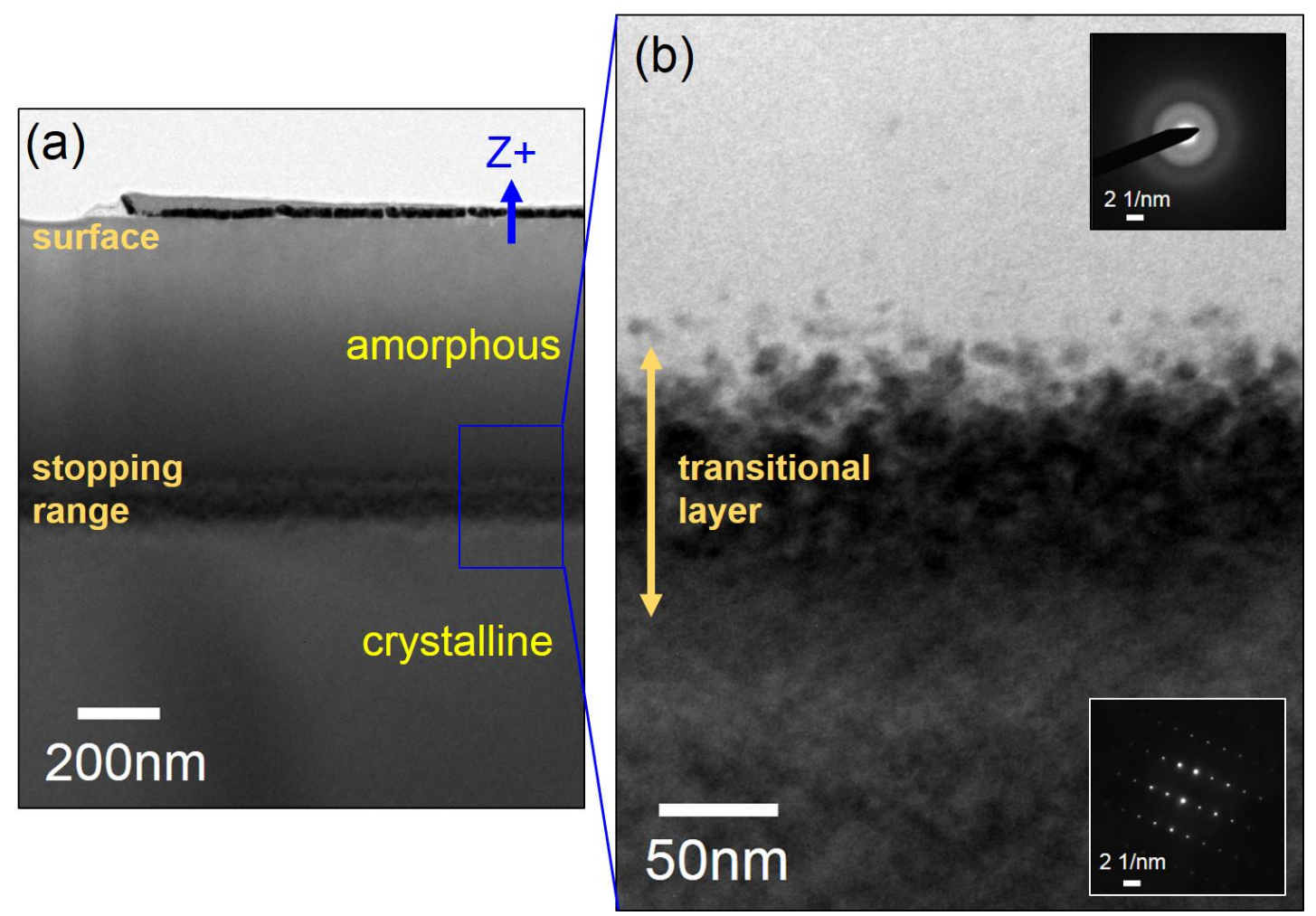

Fig. 1. Cross-sectional TEM micrographs showing the amorphous-crystalline interface as a result of high-dose $\mathrm{Ar}^{+}$irradiation. Figs. 1(a) and 1(b) were taken at 8k and 60k magnification, respectively. The insets in Fig. 1(b) are the selected area diffraction patterns (SADP). It is clear that in the surface-irradiated region, the diffraction pattern shows diffuse ring, indicating that the material is amorphous, while in the deeper unirradiated region, the diffraction shows crystalline pattern. A clear intermediate dark-contrast layer, corresponding to the argon ion stopping range and straggling, is seen.

rate for two different etching species, albeit with similar dpa values, suggests the existence of a saturation level, in which the change of etching rate with the degree of damage, as long as it is above a certain damage threshold, is minimal. In fact, Schrempel et al. showed that [3] in $\mathrm{Ar}^{+}-$ irradiated $\mathrm{X}$-cut $\mathrm{LiNbO}_{3}$, the etching rate using 3.7\% $\mathrm{HF}$ saturates when the damage reaches $\sim 0.4$ dpa. In addition, it has been found that the etching rate of this $\mathrm{Ar}^{+}$-irradiated $\mathrm{X}$-cut $\mathrm{LiNbO}_{3}$ is dependent on the acid temperature [17]. In particular, in Ref. 17, it was determined that the etching rate in such ion-irradiated sample can be increased from $\sim 20 \mathrm{~nm} / \mathrm{min}$ to $\sim 200 \mathrm{~nm} / \mathrm{min}$ 
when the temperature was raised from $24^{\circ} \mathrm{C}$ to $55^{\circ} \mathrm{C}$. Note that prior literature results also suggest that the involvement of different defect-generation mechanisms such as using light-ion or swift heavy-ion irradiation may result in somewhat different etching results. For example, an etching rate of $\sim 3.2 \pm 0.3 \mu \mathrm{m} / \mathrm{min}$ in $\mathrm{He}^{+}$-irradiated $\mathrm{LiNbO}_{3}$ after thermal activation was reported [12].

Our experiments also examined the fidelity of the transfer process in retaining the original dimensions of the etched region. Thus, for example, in the presence of significant lateral straggling, enlargement of the pattern over that of the original masked area can be expected to occur. Thus in ion patterning, loss of resolution in high-energy-particle-induced etching can be expected to be due to lateral scatter, i.e., transverse straggle, which impinges on the sidewall interface, causing undercutting. Since for ions the degree of lateral straggle is depth dependent, the magnitude of the sidewall damage or undercut will also exhibit depth dependence. More quantitatively TRIM calculations [9] show that for an $800-\mathrm{keV}-\mathrm{Ar}^{+}$irradiation in $\mathrm{LiNbO}_{3}$, corresponding to $\mathrm{R}_{\mathrm{p}} \sim 550 \mathrm{~nm}$, the depth having the most lateral straggling at the edge of the mask also occurs at the same 550-nm depth.

A series of experiments using cross-sectional SEM was used to examine this undercutting at doses above threshold; again these experiments were carried out using only heavy-ion irradiated samples. An example of one such experiment is shown in Fig. 2(a), which displays an SEM image showing the irradiated $\mathrm{LiNbO}_{3}$ after an eight-hour etching in a 5\%-HF etching solution. The figure shows clearly the profile of this undercutting, as indicated by the yellow arrow. In addition, this profile conforms well to that predicted by the damage distribution provided by TRIM, which is displayed on the right using a solid red color. The SEM micrograph images show that once the irradiated dose is above threshold, etching of this depth-dependent 
lateral damage into the masked region results in an undercut feature. The undercut reaches a maximum at $\sim 550 \mathrm{~nm}$ below the surface.

Such an undercut feature is undesirable for fabrication steps requiring sharp sidewall features such as for a channel optical waveguide or a side-wall grating structure for precision control of carrier injection [18]. One approach to mitigate such an undercut feature is to use multiple $\mathrm{Ar}^{+}$irradiation cycles [6]. In particular, if the sample is exposed to multiple irradiation steps, each having different $\mathrm{Ar}^{+}$energies, a more uniformly-distributed amorphous layer extending from the surface to the ion stopping range should be formed in the unmasked region. In order to examine the utility of this approach, we used five different $\mathrm{Ar}^{+}$irradiations performed from the highest $(800 \mathrm{keV})$ to the lowest $(75 \mathrm{keV})$ energy with the exact energy and dose of each case as described in the Experimental section above. All of these irradiation cycles were rasterscanned under water-cooled condition. After the multiple irradiation process, the photoresist mask was removed and the irradiated sample was etched in 5\% HF for eight hours in room temperature. The results of this approach are shown in the SEM cross-sectional image given in Fig. 2(b). Compared with Fig. 2(a), it is clear from Fig. 2(b) that the use of multiple irradiation yields a sharper sidewall. As predicted by TRIM [displayed on the right of Fig. 2(b)], multiple irradiation results in a more uniformly-distributed damage profile, instead of a profile having depth-dependent lateral straggling. Thus, the accompanied undercutting issue using single irradiation can be significantly suppressed. With this approach, patterning with $\sim 1 \mu \mathrm{m}$ resolution could be achieved over a large scale $\left(\sim \mathrm{cm}^{2}\right)$. Examples of the approach are displayed, using a lower magnification SEM, in Figs. 2(c) and 2(d). Note that the resolution here is mainly limited by the mask resolution created by the contact mask aligner photolithography. Sub-micrometer 
feature can in principle be fabricated in $\mathrm{LiNbO}_{3}$ as well using this method with the use of a higher resolution process using, for example, an optical stepper, for the mask fabrication.


Fig. 2. Selective etching in heavy-ion irradiated $\mathrm{LiNbO}_{3}$. In (a), a photoresist mask was created on the left side and the sample was irradiated with only single energy $(800 \mathrm{keV})$ of $\mathrm{Ar}^{+}$with a dose of $1 \times 10^{15} \mathrm{~cm}^{-2}$. Due to the higher degree of damage close to the argon-ion stopping range $\left(\mathrm{R}_{\mathrm{p}}\right)$, substantial lateral etching at $\sim \mathrm{R}_{\mathrm{p}}$ is apparent, resulting in an undercut feature (indicated by the yellow arrow). (b): Experiments showing that the undercut feature can be suppressed or ameliorated by using sequential multiple-ion-energy irradiation. (c) and (d): Demonstrations of high-resolution patterning (ion-beam lithography) in large-scale area $\left(\sim \mathrm{mm}^{2}\right)$ using this method.

Experiments were now carried out on the use of sequential irradiation followed by etching of samples using $\mathrm{He}^{+}$and $\mathrm{Fe}^{+}$ions. In the experiments, the surface was first irradiated with $3.6 \mathrm{MeV} \mathrm{He} e^{+}$to a dose of $5 \times 10^{16} \mathrm{~cm}^{-2}$. Subsequently, a photoresist mask pattern was formed on the top surface, followed by a second irradiation using a $350 \mathrm{keV} \mathrm{Fe}^{+}$beam with a dose of $1 \times 10^{15} \mathrm{~cm}^{-2}$; note that iron-ion was used in this case rather than, say $\mathrm{Ar}^{+}$or $\mathrm{Xe}^{+}$, was due to only 
for the convenience of using an existing heavy-ion source on our particular implanter. Once irradiation was completed, the resist was removed by immersion in acetone. Figure 3(a) shows an example of the optical image of the sequentially irradiated sample. Note that dislocation-line defects are clearly seen, denoted by red arrows; these lines are consistent with the same structures formed during $\mathrm{He}^{+}$etching. Thus we conclude that these lines originate from the bulkstress conditions in ion etching. In addition, the regions that were exposed to heavy-ion irradiation became darker (due to generation of color-center defects), labeled by blue arrows. After cleaning, a $\sim 250^{\circ} \mathrm{C}$ annealing cycle was performed to provide thermal activation for helium ion-slicing. Note that as reported earlier [19], annealing at $250^{\circ} \mathrm{C}$ does not fully anneal out any heavy ion-induced damage. Thus, this annealing step serves only to activate (increase) the He-
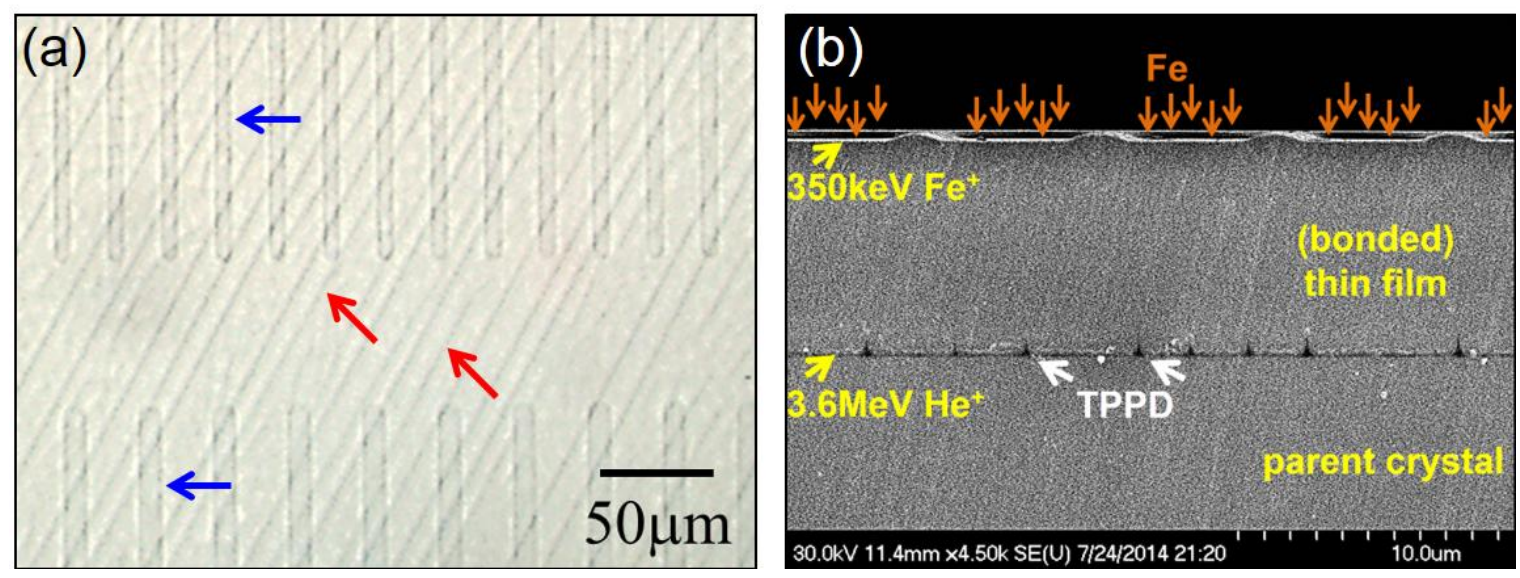

Fig. 3. Demonstration of patterning on crystal-ion-sliced thin film using sequential ion irradiation. Panel (a) shows the optical image after the sequential irradiation of $\mathrm{He}^{+}$and $\mathrm{Fe}^{+}$. Dislocation line defects, resulted from helium irradiation (labeled with red arrows) are seen. In addition, regions being iron-ion irradiated appeared darker, labeled with blue arrows. Panel (b) displays a cross-sectional SEM image after 10 min etching. It is clear that light-ion irradiation results in a localized etching and slicing profile at the ion stopping range (i.e. $\sim 9 \mu \mathrm{m}$ below the surface after $3.6 \mathrm{MeV} \mathrm{He}^{+}$), while heavy-ion irradiation gives rise to an enhanced etching in the amorphous layer starting from surface. 
induced selective etching. Finally, the activated sequentially-irradiated sample was immersed in $5 \% \mathrm{HF}$ to realize crystal ion slicing by $\mathrm{He}^{+}$and patterning by $\mathrm{Fe}^{+}$, concomitantly. To show clearly the independence of etching in the spatial regions irradiated by light and heavy ions, Fig. 3(b) shows a cross-sectional SEM image after a 10-minute wet-etching step. It is clear that after the initial 10-minute etching, enhanced localized etching occurs in the region where nuclear collisions of the light ions dominate (i.e., $\sim 9 \mu \mathrm{m}$ below the surface in our case here), while heavy-ion irradiation results in etching of the whole amorphous layer throughout the heavy-ion trajectory. Note that $\mathrm{He}^{+}$-induced thick prismatic planar defects (TPPD) were also seen [10]. The importance of this experiment is that it shows that application of the heavy-ion irradiation does not alter conditions for ion-slicing conditions. However, note that the pre-existing damage from $\mathrm{He}^{+}$has been shown in separate effects to affect the damage distribution of a successive $\mathrm{Fe}^{+}$ irradiation [19], thereby altering (reducing) the dose needed for patterning. Thus, it is important to determine the full process parameters using both ion steps and not to rely on parameters from isolated and separate steps.

In order to investigate the applicability of using such sequential-irradiation method to both structure and exfoliate a $\mathrm{LiNbO}_{3}$ thin film, as might be needed for a photonic application, features with dimensions similar to those used in sub-micrometer devices were made concomitantly with the formation of a thin self-supporting $\mathrm{LiNbO}_{3}$ film such as used in photonic crystal structures [7]. Thus, after sequential $\mathrm{He}^{+}$- and $\mathrm{Fe}^{+}$-irradiation, the sample was immersed in 5\% HF for 8-10 hours until the completion of etching. Subsequently, the patterned sliced thin film was placed in a furnace at $600^{\circ} \mathrm{C}$ under ambient conditions for 10 hours to release any irradiation-induced strain and recover from the remnant damage. The images of such freestanding structured thin film are displayed in Fig. 4. Figure 4(a) shows five fabricated grating 
patterns on a sliced $10 \mu \mathrm{m}$-thick $\mathrm{LiNbO}_{3}$ thin film, with each grating having different periods. In Fig. 4(b), higher magnification was used, showing the uniformity of the periods. Figure 4(c) is a cross-sectional image showing an etched feature. The dimensions of height $(h)$ and width $(w)$ of such etched feature are $h \sim 300 \mathrm{~nm}$ and $w \sim 600 \mathrm{~nm}$, which is a typical dimensional geometry for common high-refractive-index waveguide for photonic applications. It is clear from Figure 4 that the sequential irradiation of light and heavy ion does not affect their individual associated selective etching characteristics. Such approach thus enables easy fabrication of concomitantly patterning and slicing $\mathrm{LiNbO}_{3}$ thin film.
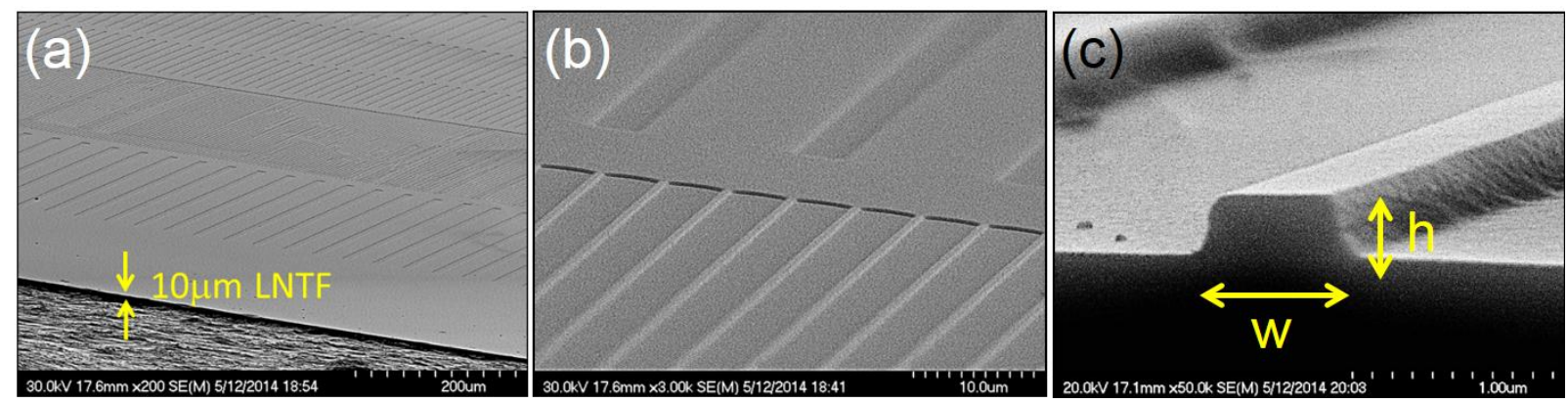

Fig. 4. (a) Patterning of an ion-sliced thin film after completion of etching in 5\% HF for 8-10 hours and subsequent annealing to release any residual strain. Panel (b) is a higher-magnification image, showing the uniformity of the grating structures with different periods. In (c), cross section of a rib-like waveguide on ionsliced freestanding thin film is demonstrated with the height and width are $h \sim 300 \mathrm{~nm}, w \sim 600 \mathrm{~nm}$.

\section{Conclusions}

In our study, the materials physics of ion-beam mediated selective etching of congruent Z-cut $\mathrm{LiNbO}_{3}$ using sequential light- and heavy-ion irradiation were investigated via electron microscopy. Ion beam scattering and the implications for etching are also discussed. It was experimentally found that different ion-induced modifications to the crystal structure give rise to different selective etching properties. Through the use of TEM and SEM, it was found that single 
heavy-ion amorphizing irradiation results in undercut feature conforming to the damage straggling distribution. With the use of multiple heavy-ion irradiation, a more uniformlydistributed amorphous layer can be created from the surface to the ion stopping range; with this approach, undesired undercut features can be suppressed. The difference between light- and heavy-ion induced selective etching process were further discussed. From the sequential irradiation experiment, we found that the presence of heavy-ion irradiation procedure has minimal effect on the condition for light-ion-induced crystal ion slicing. Through the use of sequential irradiation of light and heavy ions, their associated etching properties may be exploited to achieve easy fabrication of concomitant high-resolution patterning and exfoliation of structured freestanding $\mathrm{LiNbO}_{3}$ thin films.

\section{Acknowledgements}

This work was supported by the National Science Foundation (NSF) under Award Number ECCS-1302488. Research carried out in part at the Center for Functional

Nanomaterials, Brookhaven National Laboratory, which is supported by the U.S. Department of Energy, Office of Basic Energy Sciences, under Contract No. DE-AC02-98CH10886.

\section{Reference}

[1] W. Sohler, H. Hu, R. Ricken, V. Quiring, C. Vannahme, H. Herrmann, D. Büchter, S. Reza, W. Grundkötter, S. Orlov, H. Suche, R. Nouroozi, and Y. Min, Opt. Photonics News 19, 24 (2008).

[2] K. K. Wong, ed., Properties of Lithium Niobate, INSPEC, The Institution of Electrical Engineers, London, UK (2002).

[3] F. Schrempel, Th. Gischkat, H. Hartung, E.-B. Kley, and W. Wesch, Nucl. Instrum. Meth. B 250(1-2), 164-168 (2006).

[4] F. Nesprías, M. Venturino, M.E. Debray, J. Davidson, M. Davidson, A.J. Kreiner, D. Minsky, M. Fischer, and A. Lamagna, Nucl. Instrum. Meth. B 267(1), 69-73 (2009). 
[5] T. Gischkat, H. Hartung, F. Schrempel, E. B. Kley, A. Tünnermann, and W. Wesch, Microelectron. Engineering 86(4-6), 910-912 (2009).

[6] H. Hartung, E.-B. Kley, A. Tünnermann, T. Gischkat, F. Schrempel, and W. Wesch, Opt. Lett. 33(20), 2320-2322 (2008).

[7] R. Geiss, S. Diziain, R. Iliew, C. Etrich, H. Hartung, N. Janunts, F. Schrempel, F. Lederer, T. Pertsch, and E.-B. Kley, Appl. Phys. Lett. 97(13), 131109 (2010).

[8] D. M. Gill, D. Jacobson, C. A. White, C. D. W. Jones, Y. Shi, W. J. Minford, and A. Harris, J. Lightwave Technol. 22(3), 887-894 (2004).

[9] J. Ziegler, 2008, http://www.srim.org.

[10] A. Ofan, O. Gaathon, L. Zhang, K. Evans-Lutterodt, S. Bakhru, H. Bakhru, Y. Zhu, D. Welch, and R. M. Osgood, Jr., Phys. Rev. B 83(6), 064104 (2011).

[11] A. Ofan, L. Zhang, O. Gaathon, S. Bakhru, H. Bakhru, Y. Zhu, D. Welch, and R. M. Osgood, Jr., Phys. Rev. B 82(10), 104113 (2010).

[12] A. Ofan, O. Gaathon, L. Vanamurthy, S. Bakhru, H. Bakhru, K. Evans-Lutterodt, and R. M. Osgood Jr., Appl. Phys. Lett. 93(18), 181906 (2008).

[13] M. Levy, R. M. Osgood Jr., R. Liu, L. E. Cross, G. S. Cargill III, A. Kumar, and H. Bakhru, Appl. Phys. Lett. 73(16), 2293 (1998).

[14] D. Djukic, R. Roth, J. T. Yardley, R. M. Osgood, Jr., S. Bakhru, and H. Bakhru, Opt. Express 12(25), 6159-6164 (2004).

[15] D. Djukic, R. M. Roth, R. M. Osgood, K. Evans-Lutterodt, H. Bakhru, S. Bakhru, and D. Welch, Appl. Phys. Lett. 91(11), 112908 (2007).

[16] L.C. Feldman and J.W. Mayer, Fundamentals of Surface and Thin Film Analysis, North-Holland, New York (1986).

[17] J. Reinisch, F. Schrempel, T. Gischkat, and W. Wesch, J. Electrochem. Soc. 155(4), D298-D301 (2008).

[18] Y. Arakawa, T. Nakamura, Y. Urino, and T. Fujita, IEEE Commun. Mag. 51(3), $72-77$ (2013). 
[19] H.-C. Huang, L. Zhang, G. Malladi, J. I. Dadap, S. Manandhar, K. Kisslinger, R. S. R Vemuri, V. Shutthanandan, H. Bakhru, and R. M. Osgood, Jr, "Comparison of radiation damage by light- and heavy-ion bombardment in single-crystal $\mathrm{LiNbO}_{3}$," (in preparation). 